Case Report

\title{
Clinical and radiological deterioration due to Mycobacterium szulgai in an asthmatic patient
}

\author{
Majid Marjani ${ }^{1}$, Nahal Mansouri ${ }^{1}$, Payam Tabarsi ${ }^{1}$, Parvaneh Baghaei ${ }^{1}$, Parissa Farnia ${ }^{2}$, \\ Fatemeh-Maryam Sheikholslami ${ }^{2}$, Davood Mansouri ${ }^{1}$ \\ ${ }^{1}$ Clinical Tuberculosis and Epidemiology Research Center and ${ }^{2}$ Mycobacteriology Research Center, NRITLD, \\ Masih Daneshvari Hospital, Shahid Beheshti University of Medical Sciences, Tehran, Iran
}

\begin{abstract}
We report the case of a 23-year-old asthmatic woman who had clinical and radiological deterioration due to Mycobacterium szulgai infection and good response to anti-mycobacterial treatment without any structural abnormalities in previous lung imaging studies. M. szulgai may be considered a pathogen able to cause deterioration of respiratory health even in patients with previously normal lungs.
\end{abstract}

Key words: Mycobacterium szulgai; non tuberculous mycobacteria; asthma

J Infect Dev Ctries 2012; 6(1):89-91.

(Received 29 August 2010 - Accepted 21 February 2011)

Copyright (C) 2012 Marjani et al. This is an open-access article distributed under the Creative Commons Attribution License, which permits unrestricted use, distribution, and reproduction in any medium, provided the original work is properly cited.

\section{Introduction}

Mycobacterium szulgai was first described in 1972 [1]. It is a slow growing nontuberculous mycobacterium that may cause pulmonary or extrapulmonary disease [2]. Here we report a patient with bronchial asthma who deteriorated because of a M. szulgai infection with subsequent good clinical and radiological response to anti-mycobacterial drugs.

\section{Case report}

A 23-year-old woman was referred with complaints of dyspnea and increased productive cough. She was known to have had bronchial asthma for three years and had been treated with different types of medications, particularly systemic steroids.

One year ago, her chest X-ray and lung computed tomography (CT) scan were normal. Two months ago, she was admitted to another center where she was diagnosed of allergic bronchopulmonary aspergillosis (ABPA) because of a moderate elevation of $\mathrm{IgE}(450 \mathrm{IU} / \mathrm{ml})$, with no other positive findings; diagnostic criteria for diagnosis of ABPA were not fulfilled. She had been treated with prednisolone $25 \mathrm{mg}$ per day combined with inhaled Ipratropium, Fluticasone and Salmeterol, with no improvement.
The patient was a pharmacy student without any history of sick contact, recent travel, smoking, or drug abuse. Except for asthma, she had no other significant medical history.

On admission to the hospital, the patient was dyspneic, afebrile, and had generalized wheezing. Chest X ray and lung CT scan (Figures A and B) revealed multiple bronchiectatic lesions associated with nodules.

Peripheral blood leukocyte count, hemoglobin, and platelet count were normal with normal differentiation. The erythrocyte sedimentation rate was $42 \mathrm{~mm} / \mathrm{h}$. All biochemical test results were normal. Specific serum IgG and IgE for Aspergillus and HIV serology were negative.

No bacterial or fungal agents were found on sputum and bronchoalveolar lavage (BAL) fluid but one sample each of the sputum and BAL fluid were positive for acid fast bacilli. Although the sputum and BAL fluid cultures remained negative for six months after incubation, Mycobacterium szulgai was identified by PCR from both specimens. For this purpose a $440 \mathrm{bp}$ fragment of the $65-\mathrm{kDa}$ heat shock protein gene (hsp65) was amplified using two oligonucleotide primers (TB11 and TB12) as described by Telenti et al. [3]. Then the PCR product was digested by two endonuclease enzymes, BstEII and HaeIII (fermentase) and subjected to 
Figure 1. Radiographic images of the patient. Chest radiograph (A) and chest computed tomography (B) on admission compared with chest radiograph (C) and chest computed tomography (D) four months after treatment.

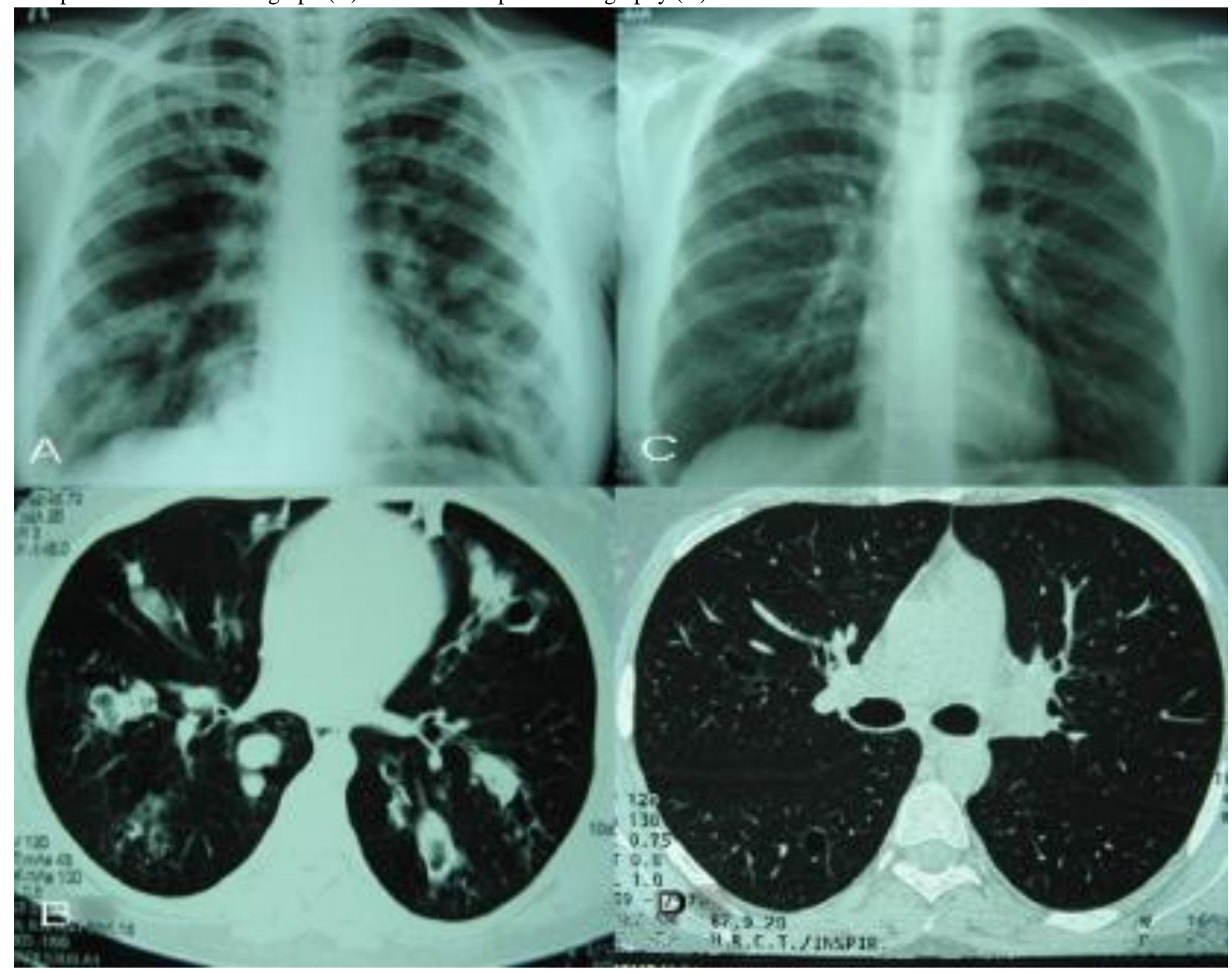

polyacrylamide gel electrophoresis (PAGE) for restriction fragment length polymorphism (RFLP) analysis. The pattern obtained was compared with that of standard Institut Pasteur mycobacterium strains [4].

The patient was treated with a combination of clarithromycin (500 mg every 12 hours), ethambutol (1,000 mg daily), and ofloxacin (300 mg every 12 hours) with significant improvement of clinical symptoms. She was free of cough and dyspnea despite discontinuation of systemic and inhaled steroids within two months after treatment. Four months later, a chest $\mathrm{X}$ ray (figure $\mathrm{C}$ ) was reported to be normal and lung CT demonstrated significant reduction of nodules and bronchiectatic lesions in terms of number and size (Figure D). The treatment was continued for 18 months and the patient remains healthy more than one year after termination of treatment.

\section{Discussion}

Obstructive lung disease, cigarette smoking, alcoholism, and systemic impaired immunity are risk factors of Mycobacterium szulgai infection [5]. It is a rare cause of infection and difficult to identify [6], but recovery of $M$. szulgai from clinical specimens is highly indicative of disease, regardless of the 
American Thoracic Society criteria for diagnosis of nontuberculous mycobacterial disease $[2,7,8]$.

Despite the lack of standard recommendations, a regimen consisting of three drugs is reported to guarantee a low relapse rate [6]. Some authorities suggest isoniazid, rifampin, and ethambutol with or without streptomycin or pyrazinamide for at least 18 months or 12 months after sputum culture conversion $[7,9]$, although others have found favorable outcomes with a 12-month regimen of rifampin, ethambutol, and clarithromycin [2].

Although we didn't have any positive cultures, the positive smear, PCR, and appropriate response to treatment are most likely to be compatible with the diagnosis of $M$. szulgai infection. The extent of pulmonary lesions may also be related to inappropriate systemic steroid use.

To our knowledge, this is the first report of $M$. szulgai infection from Iran. The interesting aspect of this case is the absence of any structural abnormalities in previous lung imaging studies and the occurrence of new extensive lesions which were attributable to $M$. szulgai infection, which resolved nearly completely after treatment.

This finding is intriguing as most other nontuberculous mycobacteria usually infect patients with immune deficiency, bronchiectatic changes, or other abnormalities in lungs.

We conclude that $M$. szulgai may be considered a pathogen able to cause deterioration of respiratory health even in patients with previously normal lungs.

\section{References}

1. Marks J, Jenkins PA, Tsukamura M (1972) Mycobacterium szulgai a new pathogen. Tubercle 53: 210-214.

2. van Ingen $J$, Boeree MJ, de Lange WC, de Haas PE, Dekhuijzen PN, van Soolingen D (2008) Clinical relevance of Mycobacterium szulgai in the Netherlands. Clinical Infectious Diseases 46: 1200-1205.
3. Telenti A, Marchesi F, Balz M, Bally F, Böttger EC, Bodmer T (1993) Rapid identification of mycobacteria to the species level by polymerase chain reaction and restriction enzyme analysis. $\mathbf{J}$ Clin Microbiol 31: 175-178.

4. Brunello F, Ligozzi M, Cristelli E, Bonora S, Tortoli E, Fontana R (2001) Identification of 54 mycobacterial species by PCR-restriction fragment length polymorphism analysis of the hsp65 gene. J Clin Microbiol 39: 2799-2806.

5. Gutiérrez M, Feola M, Lenge L, Rey R, Hoffman M (2007) First pulmonary case reported in Argentina of infection with Mycobacterium szulgai, a rare pathogen. J Clin Microbiol 45: 3121-3124.

6. Tortoli E, Besozzi G, Lacchini C, Penati V, Simonetti MT, Emler S (1988) Pulmonary infection due to Mycobacterium szulgai: case report and review of the literature. Eur Respir J 11: 975-977.

7. Piersimoni C, Scarparo C (2008) Pulmonary infections associated with non- tuberculous mycobacteria in immunocompetent patients. Lancet Infect Dis 8: 323-334.

8. Griffith DE, Aksamit T, Brown-Elliott BA, Catanzaro A, Daley C, Gordin F, Holland SM, Horsburgh R, Huitt G, Iademarco MF, Iseman M, Olivier K, Ruoss S, von Reyn CF, Wallace RJ Jr, Winthrop K; ATS Mycobacterial Diseases Subcommittee; American Thoracic Society; Infectious Disease Society of America (2007) An official ATS/IDSA statement: diagnosis, treatment, and prevention of nontuberculous mycobacterial diseases. Am J Respir Crit Care Med 175: 367416.

9. Glassroth J (2008) Pulmonary disease due to nontuberculous Mycobacteria. Chest 133: 243-251.

\section{Corresponding author}

Dr. Davood Mansouri

Department of Internal Medicine

Division of Infectious Diseases and Clinical Immunology

National Research Institute of Tuberculosis and Lung Disease

Masih Daneshvari Hospital

Shaheed Beheshti University of Medical Sciences

Tehran, Iran 1955841452

Telephone: +98-21-26109590

Fax: +98-21-26105050

Email: dmansouree@yahoo.com

Conflict of interests: No conflict of interests is declared. 